\title{
CONGRUENCE-FREE REGULAR SEMIGROUPS
}

\author{
by W. D. MUNN
}

(Received 11th May 1984)

A semigroup is said to be congruence-free if and only if its only congruences are the universal relation and the identical relation. Congruence-free inverse semigroups were studied by Baird [2], Trotter [19], Munn [15,16] and Reilly [18]. In addition, results on congruence-free regular semigroups have been obtained by Trotter [20], Hall [4] and Howie [7].

It was shown in [15] that if $E$ is the semilattice of a congruence-free inverse semigroup, other than a simple group, then there is a congruence-free inverse semigroup $T_{E}^{*}$ with semilattice (isomorphic to) $E$ and such that each congruence-free inverse semigroup with semilattice $E$ is isomorphic to a full inverse subsemigroup of $T_{E}^{*}$. For $E$ as above, $T_{E}^{*}$ can thus loosely be described as the "greatest" congruence-free inverse semigroup with semilattice $E$. The main purpose of the present paper is to extend this result from inverse semigroups to regular semigroups. Here we replace semilattices by regular biordered sets, as defined by Nambooripad [17], and make use of his work (equivalent to that of Hall [3]) on the analogue for regular semigroups of the inverse semigroup $T_{E}$ introduced by the author in [13].

The first section provides a brief review of some properties of group congruences on a regular semigroup $S$ and of the "hypercore" of $S$, a concept introduced by T. E. Hall and the author in [5]. In the second section, for a regular biordered set $E$, we define $T_{E}^{*}$ to the hypercore of Nambooripad's semigroup $T_{E}$ [17]. It is shown that if $E$ is the biordered set of idempotents of a congruence-free regular semigroup which is not a simple group then $T_{E}^{*}$ is a congruence-free regular semigroup with $E$ as its biordered set of idempotents (to within isomorphism) and that each congruence-free regular semigroup with this biordered set of idempotents is isomorphic to a full regular subsemigroup of $T_{E}^{*}$. An example is given in the third section to show that there need not exist a "least" congruence-free inverse semigroup with semilattice $E$, where $E$ is the semilattice of some congruence-free inverse semigroup. The paper ends with a short fourth section which provides sufficient conditions for $A$ to be congruence-free, given that $S$ and $A$ are full regular subsemigroups of a regular semigroup $T$ with $S \subseteq A \subseteq T$, that $S$ and $T$ are themselves congruence-free, and that $T$ is not a simple group.

The principal results were announced by the author at a conference on semigroups held at Oberwolfach, W. Germany, in May 1981. In the version presented there, the hypercore of a regular semigroup was defined as the limit of its core series (see $[5$, Theorem 3]). 


\section{Preliminaries}

The notation and terminology is based on that of Howie [6]. In particular, the set of idempotents of a semigroup $S$ will be denoted by $E(S)$, the set of all inverses in $S$ of an element $a$ of $S$ by $V_{S}(a)$, and the subsemigroup generated by a nonempty subset $A$ of $S$ by $\langle A\rangle$. By the greatest [least] subsemigroup of $S$ with a given property $\mathscr{P}$ we mean a subsemigroup $A$ with property $\mathscr{P}$ such that $A \supseteq B[A \subseteq B]$ for all subsemigroups $B$ with property $\mathscr{P}$. If such an $A$ exists then it is, of course, unique.

Now let $S$ be a regular semigroup. It is well known and readily verified that a congruence $\rho$ on $S$ is a group congruence (that is, $S / \rho$ is a group) if and only if $\rho \supseteq E(S) \times E(S)$. From this it follows that $S$ possesses a least group congruence $\sigma(S)$, namely the congruence generated by $E(S) \times E(S)$ [10]. As in [5], the $\sigma(S)$-class containing $E(S)$ will be called the core of $S$ and denoted by core $(S)$. A subsemigroup $T$ of $S$ is termed

(i) full if and only if $E(S) \subseteq T$,

(ii) unitary if and only if

$$
(\forall t \in T)(\forall x \in S) \quad[t x \in T \Rightarrow x \in T] \text { and }[x t \in T \Rightarrow x \in T] \text {, }
$$

(iii) self-conjugate if and only if

$$
(\forall t \in T)(\forall x \in S) \quad\left(\forall x^{\prime} \in V_{S}(x)\right) \quad x^{\prime} t x \in T .
$$

As is easily seen, there is a least self-conjugate subsemigroup of $S$ containing a given subsemigroup $T$ of $S$. It will be denoted by $T^{S}$. Thus $S$ has a least full self-conjugate subsemigroup, namely $\langle E(S)\rangle^{\mathbf{S}}$. Similarly, $S$ has a least full unitary self-conjugate subsemigroup and, in [8], Feigenbaum showed that this coincides with core $(S)$. Evidently $\langle E(S)\rangle^{S} \subseteq$ core $(S)$.

The following lemma [8; 9, Corollary 2.2] provides a characterisation of $\sigma(S)$ in terms of the least full self-conjugate subsemigroup of $S$. It generalises an earlier result $[12 ; 6$, Theorem V.3.1] on inverse semigroups.

Lemma 1. Let $S$ be a regular semigroup. Then, for all $a, b \in S$,

$$
(a, b) \in \sigma(S) \quad \Leftrightarrow \quad\left(\exists u, v \in\langle E(S)\rangle^{S}\right) a u=v b
$$

In [5], T. E. Hall and the author introduced the concept of the hypercore, hyp (S), of a semigroup $S$. Let $\mathscr{S}_{S}$ denote the set of all subsemigroups $A$ of $S$ with no non-universal congruence $\rho$ such that $A / \rho$ is cancellative. Then, assuming that $\mathscr{S}_{S} \neq \varnothing$ (which is certainly true if $E(S) \neq \varnothing)$, hyp $(S)$ is the subsemigroup of $S$ generated by the union of all $A$ in $\mathscr{S}_{S}$. From [5, Theorems 1 and 2] we obtain a result that is the key to the developments in Section 2 below:

Lemma 2. Let $S$ be a regular semigroup. Then hyp $(S)$ is a full unitary subsemigroup of $S$ contained in core $(S)$ and is the greatest regular subsemigroup $A$ of $S$ such that $\operatorname{core}(A)=A$.

Another description of the hypercore of a regular semigroup is given in [5, Theorem 3$]$. 


\section{The semigroup $T_{E}^{*}$}

Recall that a semigroup $S$ is termed "fundamental" if and only if the only congruence on $S$ contained in $\mathscr{H}$ is the identity. For $S$ regular, $S$ is fundamental if and only if it has no idempotent-separating congruence other than the identity congruence $[10 ; 6$, Proposition II.4.8].

For ease of reference we restate part of $[3$, Corollary 6] at this stage.

Lemma 3. Every full regular subsemigroup of a fundamental regular semigroup is fundamental.

The set of idempotents of a regular semigroup is a regular biordered set, in the sense of Nambooripad [17]. Now let $E$ be a regular biordered set. Then there exists a regular semigroup $T_{E}$ with the following properties ([17]; see also Hall's earlier paper [3] for an equivalent result):

(i) $E\left(T_{E}\right) \cong E$ (qua biordered sets);

(ii) $T_{E}$ is fundamental;

(iii) if $S$ is a regular semigroup and $E=E(S)$ then there is a homomorphism $\theta: S \rightarrow T_{E}$ such that $E \theta=E\left(T_{E}\right)$ and $\theta \circ \theta^{-1} \subseteq \mathscr{H}$.

We denote hyp $\left(T_{E}\right)$ by $T_{E}^{*}$.

If $E$ is a semilattice then $T_{E}$ and $T_{E}^{*}$ coincide with the similarly-designated semigroups introduced by the author in [13] and [14]. (It should be noted that in [17] the notation $T_{E}^{*}$ is used for an altogether different concept.)

We say that a regular semigroup $S$ can be fully embedded in a regular semigroup $T$ if and only if there is an injective homomorphism $\theta: S \rightarrow T$ such that $S \theta$ is a full subsemigroup of $T$.

Theorem 1. Let $E$ be a regular biordered set.

(i) $T_{E}^{*}$ is a fundamental full unitary regular subsemigroup of $T_{E}$ and $\operatorname{core}\left(T_{E}^{*}\right)=T_{E}^{*}$.

(ii) If $S$ is a fundamental regular semigroup with $E(S) \cong E$ and $\operatorname{core}(S)=S$ then $S$ can be fully embedded in $T_{E}^{*}$.

Proof. (i) By Lemma 2, $T_{E}^{*}$ is a full unitary regular subsemigroup of $T_{E}$ and core $\left(T_{E}^{*}\right)=$ $T_{E}^{*}$. But $T_{E}$ is fundamental and so, by Lemma $3, T_{E}^{*}$ is fundamental.

(ii) Let $S$ be a fundamental regular semigroup with $E(S) \cong E$ and $\operatorname{core}(S)=S$. Then there exists a homomorphism $\theta: S \rightarrow T_{E}$ such that (a) $E(S) \theta=E\left(T_{E}\right)$ and (b) $\theta \circ \theta^{-1} \subseteq \mathscr{H}$. Evidently $S \theta$ is a regular subsemigroup of $T_{E}$. Moreover, by (b), since $S$ is fundamental, $\theta$ is injective. Hence $\operatorname{core}(S \theta)=S \theta$ and so, by Lemma 2, $S \theta \subseteq T_{E}^{*}$. Also, by (a), $E(S \theta)=$ $E\left(T_{E}\right)=E\left(T_{E}^{*}\right)$.

The principal result of this section (Theorem 2) will be obtained by combining Theorem 1 with the following lemma. 
Lemma 4. Let $A$ be a regular semigroup such that

(i) $A$ is fundamental,

(ii) $\operatorname{core}(A)=A$,

(iii) $A$ contains a congruence-free full regular subsemigroup.

Then $A$ is congruence-free.

Proof. Let $\rho$ be a congruence on $A$ other than the identity congruence. Since $A$ is fundamental there exist $e, f \in E(A)$ such that $(e, f) \in \rho$ and $e \neq f$. By hypothesis, $A$ contains a congruence-free full regular subsemigroup $B$. Write $\tau=\rho \cap(B \times B)$. Then $\tau$ is a congruence on $B$; also $\tau$ is not the identity congruence, since it contains $(e, f)$. Consequently $\tau=B \times B$ and so $B \times B \subseteq \rho$. In particular, $E(A) \times E(A) \subseteq \rho$, which shows that $\rho$ is a group congruence. But $\operatorname{core}(A)=A$; that is $\sigma(A)=A \times A$. Hence $\rho=A \times A$. Thus $A$ is congruence-free.

Theorem 2. Let $S$ be a congruence-free regular semigroup which is not a simple group and let $E$ denote $E(S)$. Then $T_{E}^{*}$ is a congruence-free regular semigroup and $S$ can be fully embedded in $T_{E}^{*}$.

Proof. By Theorem 1(i), $T_{E}^{*}$ is a fundamental regular semigroup with core $\left(T_{E}^{*}\right)=T_{E}^{*}$. Since $S$ is not a group, $|E|>1$. Hence $S$ is fundamental and $\operatorname{core}(S)=S$. Thus, by Theorem 1(ii), $S$ can be fully embedded in $T_{E}^{*}$. The hypotheses of Lemma 4 are therefore satisfied when $A=T_{E}^{*}$ and so $T_{E}^{*}$ is congruence-free.

This theorem generalises an earlier result on inverse semigroups [15, Corollary 1.8]. Note that if $S=S^{0}$ above then $T_{E}^{*}$ coincides with $T_{E}$.

\section{A counterexample to a conjecture}

Let $E$ be the regular biordered set of idempotents of some congruence-free regular semigroup which is not a simple group. Theorem 2 can be interpreted as showing that there is a greatest congruence-free regular semigroup $S$ with $E(S)=E$, namely $T_{E}^{*}$. It is natural to conjecture that there is also a least such semigroup.

For certain choices of $E$ a least congruence-free regular semigroup $S$ with $E(S)=E$ does indeed exist. It can be shown, for instance, that if $S$ is a congruence-free (or, more generally, fundamental) completely 0 -simple semigroup then $S \cong T_{E(S)}$ and so $S$ is uniquely determined by $E(S)$. Again, consider a congruence-free idempotent-generated regular semigroup $S$. (Examples of such semigroups have been given by Howie [7]; see also Hall [4].) Let $E=E(S)$. Then, since $S$ can be fully embedded in $T_{E}^{*}, S \cong\left\langle E\left(T_{E}^{*}\right)\right\rangle$. Now let $A$ be any congruence-free regular semigroup with $E(A) \cong E$. Then $A$ is isomorphic to a full regular subsemigroup $A^{\prime}$ of $\mathrm{T}_{E}^{*}$ and hence $\left\langle E\left(T_{E}^{*}\right)\right\rangle \subseteq A^{\prime}$. Thus $S$ can be regarded as the least congruence-free regular semigroup with regular biordered set $E$.

The conjecture is false, however, even in the inverse case, as will be demonstrated by the example below. First, we recall some definitions $[2,14]$ concerning a semilattice $E=E^{0}$. We say that $E$ is disjunctive if and only if for all $e, f \in E$ with $e \neq f$ there exists 
$g \in E$ such that exactly one of $e g$ and $f g$ is 0 . The domain and codomain of an element $\alpha$ of the inverse semigroup $T_{E}$ will be denoted by $\Delta(\alpha)$ and $\nabla(\alpha)$ respectively. An inverse subsemigroup $A$ of $T_{E}$ is said to be 0 -subtransitive if and only if for all $e, f \in E \backslash 0$ there exists $\alpha \in A$ such that $\Delta(\alpha)=E e$ and $\nabla(\alpha) \subseteq E f$. Further, $E$ is termed 0 -subuniform if and only if $T_{E}$ is itself 0 -subtransitive. Baird [2] and Trotter [19] have shown that $E$ is the semilattice of a congruence-free inverse semigroup (with zero) if and only if $E$ is 0 -subuniform and disjunctive; moreover, if $S$ is any congruence-free inverse semigroup with semilattice $E$ then $S$ is isomorphic to a 0 -subtransitive inverse subsemigroup of $T_{E}$ and every 0 -subtransitive inverse subsemigroup of $T_{E}$ (including, of course, $T_{E}$ itself) is congruence-free. Some analogous results for congruence-free regular semigroups with zero were subsequently obtained by Trotter [20].

Example. Let $F_{2}$ denote the free monoid generated by two symbols $a, b$ : thus $F_{2}$ consists of all words in $a, b$ together with the empty word 1 . The length of a word $w \in F_{2}$ will be denoted by $l(w)$. We define a partial order $\leqq$ on $F_{2}$ by

$$
u \leqq v \Leftrightarrow v \text { is an initial segment of } u
$$

and extend it to a partial order $\leqq$ of $F_{2}^{0}$ by requiring that $0 \leqq u$ for all $u \in F_{2}^{0}$. Then $\left(F_{2}^{0}, \leqq\right)$ is a lower semilattice, with least element 0 and greatest element 1 . We denote it by $E$. The principal ideal of $E$ generated by $w \in F_{2}$ will be written as [w]. As noted by Baird [2], $E$ is 0 -subuniform and disjunctive; thus $T_{E}$ is a congruence-free inverse semigroup with zero.

To show that there is no least congruence-free inverse semigroup with semilattice $E$ it suffices to show that $T_{E}$ contains no least 0 -subtransitive inverse subsemigroup. Suppose that $T_{E}$ does contain a least such subsemigroup $S$. Choose $\gamma \in S$ such that $\Delta(\gamma)=E$ and $\nabla(\gamma) \neq E$. Then $\nabla(\gamma)=[w]$ for some $w \neq 1$. Let $n=l(w)+1$ and let $u_{1}, u_{2}, \ldots, u_{2^{n}}$ denote the elements of $F_{2}$ of length $n$. Now define $\alpha_{i} \in T_{E}\left(i=1,2, \ldots, 2^{n}\right)$ by

$$
\Delta\left(\alpha_{i}\right)=E, \quad \nabla\left(\alpha_{i}\right)=\left[u_{i}\right], \quad x \alpha_{i}= \begin{cases}u_{i} x & \text { if } x \in F_{2} \\ 0 & \text { if } x=0\end{cases}
$$

and let $A$ denote the inverse subsemigroup of $T_{E}$ generated by $E\left(T_{E}\right)$ and the elements $\alpha_{1}, \alpha_{2}, \ldots, \alpha_{2 n}$. Note that, for all $\alpha \in A \backslash 0$ and all $x \in \Delta(\alpha) \backslash 0$,

$$
l(x) \equiv l(x \alpha) \quad(\bmod n) .
$$

Hence, in particular, $\gamma \notin A$ : for $1 \in \Delta(\gamma) \backslash 0, l(1)=0$ and $l(1 \gamma)=l(w)=n-1>0$.

We now show that $A$ is 0 -subtransitive. Let $p, q \in E \backslash 0\left(=F_{2}\right)$. Choose $r \in F_{2}$ such that $l(q r) \equiv 0(\bmod n)$. Then there exist $m \in \mathbb{N}$ and $i_{1}, i_{2}, \ldots, i_{m} \in\left\{1,2, \ldots, 2^{n}\right\}$ such that $q r=$ $u_{i_{1}} u_{i_{2}} \ldots u_{i_{m}}$. Hence $1 \alpha_{i_{m}} \alpha_{i_{m-1}} \ldots \alpha_{i_{1}}=q r$. Let $\varepsilon_{p}$ denote the identity mapping on [p] and write $\beta=\varepsilon_{p} \alpha_{i_{m}} \alpha_{i_{m-1}} \ldots \alpha_{i_{1}}$. Evidently $\beta \in A$ and

$$
\Delta(\beta)=[p], \quad \nabla(\beta)=[q r p] \subseteq[q] .
$$


Thus $A$ is 0 -subtransitive. But $\gamma \notin A$ and so $S \nsubseteq A$. This contradicts our assumption that $S$ is the least 0 -subtransitive inverse subsemigroup of $T_{E}$. Hence no such subsemigroup exists.

\section{A convexity question}

In this section we concern ourselves with the following question. Suppose that $T$ is a congruence-free regular semigroup, other than a simple group, and that $S, A$ are full regular subsemigroups with $S \subseteq A \subseteq T$ and $S$ congruence-free. Under what conditions is $A$ congruence-free?

We show that $A$ is always congruence-free when $T=T^{0}$ and provide a sufficient condition for this to hold when $T \neq T^{0}$.

Theorem 3. Let $T$ be a congruence-free regular semigroup, other than a simple group, and let $S, A$ be full regular subsemigroups such that

(i) $S \subseteq A \subseteq T$,

(ii) $S$ is congruence-free,

(iii) $\langle E\rangle^{S}=\langle E\rangle^{T}$ if $T \neq T^{0}$, where $E=E(S)=E(T)$.

Then $A$ is congruence-free.

Proof. Since $T$ is fundamental, so also is $A$, by Lemma 3 . We proceed to show that core $(A)=A$. This is immediate if $T=T^{0}$; for then $A=A^{0}$. Hence suppose that $T \neq T^{0}$.

Note first that $\langle E\rangle^{S}=\bigcup_{i=1}^{\infty} S_{i}$, where $S_{1}=\langle E\rangle$ and, for $i \in \mathbb{N}, S_{i+1}$ is defined inductively as the subsemigroup of $S$ generated by all elements of the form $x^{\prime} a x$, with $a \in S_{i}, x \in S^{1}$ and $x^{\prime} \in V_{S^{1}}(x)$ [11, p. 493]. Using this, and the corresponding results for $A$ and $T$, we see from (i) that $\langle E\rangle^{S} \subseteq\langle E\rangle^{A} \subseteq\langle E\rangle^{T}$. Hence, by (iii), $\langle E\rangle^{A}=\langle E\rangle^{T}$. Now let $(a, b) \in A \times A$. Then, since core $(T)=T$, we have that $(a, b) \in \sigma(T)$ and so, by Lemma 1 , there exist $u, v \in\langle E\rangle^{T}$ such that $a u=v b$. Since $\langle E\rangle^{A}=\langle E\rangle^{T}$ it follows from Lemma 1 that $(a, b) \in \sigma(A)$. Thus $\sigma(A)=A \times A$; that is, $\operatorname{core}(A)=A$.

The result now follows from Lemma 4.

It is easily verified that if, in addition, $S$ is [0-]bisimple then $A$ is [0-]bisimple.

Since condition (iii) is automatically satisfied if $T$ is an inverse semigroup $\left(\langle E\rangle^{T}\right.$ then reducing to $E$ ), we obtain the following corollary - which can also be deduced from the results in [15, Section 1].

Corollary. Let $T$ be a congruence-free inverse semigroup, other than a simple group, and let $S, A$ be full inverse subsemigroups such that

(i) $S \subseteq A \subseteq T$,

(ii) $S$ is congruence-free.

Then $A$ is congruence-free.

Finally, we remark that nothing new arises if we replace the adjective "inverse" by "orthodox" in the hypotheses of the corollary: for, as shown by Bailes [1, p. 498], every congruence-free orthodox semigroup $S$ with $|S|>2$ is an inverse semigroup. 


\section{REFERENCES}

1. G. L. Balles, JR., Right inverse semigroups, J. Algebra 26 (1973), 492-507.

2. G. R. BAIRD, Congruence-free inverse semigroups with zero, J. Austral. Math. Soc. (A) 20 (1975), 110-114.

3. T. E. Hall, On regular semigroups, J. Algebra 24 (1973), 1-24.

4. T. E. Hall, Amalgamation and inverse and regular semigroups: a brief survey, Proc. conference on regular semigroups (Northern Illinois University, 1979).

5. T. E. Hall and W. D. MunN, The hypercore of a semigroup, Proc. Edinburgh Math. Soc. 28 (1985), 107-112.

6. J. M. Howie, An introduction to semigroup theory (Academic Press, New York, 1976).

7. J. M. Howie, A class of bisimple, idempotent-generated congruence-free semigroups, Proc. Roy. Soc. Edinburgh (A) 88 (1981), 169-184.

8. R. Feigenaaum, Kernels of regular semigroup homomorphisms (Ph.D. dissertation, University of South Carolina, 1975).

9. R. Feigenbaum, Regular semigroup congruences, Semigroup Forum 17 (1979), 373-377.

10. G. Lallement, Congruences et équivalences de Green sur un demi-groupe régulier, $C . R$. Acad. Sci. Paris Sér. A 262 (1966), 613-616.

11. D. B. McAlister, Regular semigroups, fundamental semigroups and groups, J. Austral. Math. Soc. (A) 29 (1980), 475-503.

12. W. D. MunN, A class of irreducible matrix representations of an arbitrary inverse semigroup, Proc. Glasgow Math. Assoc. 5 (1961), 41-48.

13. W. D. MunN, Uniform semilattices and bisimple inverse semigroups, Quart. J. Math. (Oxford) (2) 17 (1966), 151-159.

14. W. D. Munn, Fundamental inverse semigroups, Quart. J. Math. (Oxford) (2) 21 (1970), $157-170$.

15. W. D. MunN, Congruence-free inverse semigroups, Quart. J. Math. (Oxford) (2) 25 (1974), 463-484.

16. W. D. MunN, A note on congruence-free inverse semigroups, Quart. J. Math. (Oxford) (2) 26 (1975), 385-387.

17. K. S. S. Nambooripad, Structure of regular semigroups I, Mem. Amer. Math. Soc. 224 (1979).

18. N. R. Reilly, Congruence-free semigroups, Proc. London Math. Soc. (3) 33 (1976), $497-514$.

19. P. G. Trotter, Congruence-free inverse semigroups, Semigroup Forum 9 (1974), 109-1 16. $1-5$.

20. P. G. Trotter, Congruence-free regular semigroups with zero, Semigroup Forum 12 (1976),

Department of Mathematics

UNIVERSITY OF GLASGOW

GLASGOW G12 8QW

SCOTLAND 